Journal of

Molecular Microbiology

and Biotechnology

\title{
Desalted Deep Sea Water Increases Transformation and Homologous Recombination Efficiencies in Dictyostelium discoideum
}

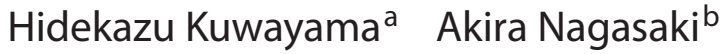 \\ ${ }^{a}$ Graduate School of Life and Environmental Sciences, University of Tsukuba, and ${ }^{b}$ Research Institute for \\ Cell Engineering, National Institute of Advanced Industrial Science and Technology, Tsukuba, Ibaraki, Japan
}

\section{Key Words}

Dictyostelium discoideum • Deep sea water •

Transformation · Homologous recombination · Growth medium

\begin{abstract}
The life cycle of Dictyostelium discoideum consists of many cellular and developmental aspects. By virtue of its relatively high transformation efficiency and a small haploid genome, this organism has proven to be advantageous for characterizing gene functions. However, a much higher transformation efficiency is required as one of the prerequisites for unraveling gene function on a genome-wide scale. In this study, we describe the positive effect of desalted deep sea water, when used as a solvent medium, on the transformation and homologous recombination efficiencies in Dictyostelium. A standard Dictyostelium medium HL5 containing desalted deep sea water, HL5dsw, distinctly increased both the transformation and homologous recombination efficiencies by approximately 2- to 3-fold. Furthermore, we observed that the growth of cells in HL5dsw both before and after electroporation contributed to the increase in transformation efficiency. These results indicate that a simple modification of the solvent medium remarkably enhanced the isolation of transformants and gene-targeted clones, which had previously been difficult to isolate.
\end{abstract}

Copyright $\odot 2007$ S. Karger AG, Basel
(C) 2007 S. Karger AG, Basel

$1464-1801 / 08 / 0144-0157 \$ 24.50 / 0$

Fax +4161306 1234

E-Mail karger@karger.ch

www.karger.com
Accessible online at:

www.karger.com $/ \mathrm{mmb}$

\section{Introduction}

Dictyostelium discoideum is a solitary amoeba and develops into a multicellular fruiting body that consists of two distinct types of differentiated cells: the stalk and the spore [Kessin, 2001]. In Dictyostelium, transformation is a standard technique for investigating gene function [Knecht and Pang, 1995]. The recent completion of the sequencing of its genome has accelerated the molecular genetical approach using this microorganism [Eichinger et al., 2005]. Generally, the transformation efficiency has been of relatively minor importance in most experiments because only a few clones are sufficient for the necessary analysis. However, for the largescale isolation of primary transformants, as in the case of random selection of gene-tagging mutation by random restriction enzyme-mediated integration [Kuspa and Loomis, 1992], and for the complementation of a mutant strain, a high yield of independent transformants is indispensable. It has been reported that Dictyostelium cells exhibit a homologous recombination efficiency that is sufficiently high to ensure simultaneous homologous recombination of multiple genes [Betapudi et al., 2004]. However, during certain experiments for gene disruption, many researchers have occasionally observed that even a single gene-disrupted cell can be difficult to isolate, presumably due to the low frequency of homologous recombination in the targeted gene. In 
this case, it becomes very difficult to decipher whether the gene disruption will end in the death of the cell or whether the isolation of gene-disrupted cells can be achieved by raising the yield of transformation.

Many attempts have been made to improve the yield of transformants. Cells in S or early G2 phases in the cell cycle have higher transformation competence than in the other phases of the cell cycle [Nellen and Saur, 1988]. The oscillating electroporation technique was compared experimentally to exponential decay electroporation, and was demonstrated to result in a 20 -fold increase in the efficiency of transformation [Alibaud et al., 2003]. However, these approaches did not yield any significant increase in the homologous recombination efficiency.

Previously, it was reported that DNA binds natural clay minerals and that this facilitates the transformation of DNA in a microorganism [Khanna and Stotzky, 1992]. Interestingly, Khanna and Stotzky demonstrated that natural minerals increase the resistance of bound DNA to degradation by DNase I. In this study, we focused on the use of deep sea water (DSW) as a natural solvent and demonstrated that the modification of the solvent medium from ultrapure water to desalted DSW effected substantial improvements in both transformation and homologous recombination efficiencies. This is probably due to the minor substances dissolved in desalted DSW.

\section{Results}

\section{Increase in Transformation Efficiency in DSW}

Medium

In order to improve transformation conditions, we focused on investigating the use of desalted DSW instead of our usual solvent medium - ultrapure water - for the process of transforming Dictyostelium cells. The transformation was carried out using the standard electroporation technique described in Experimental Procedures. The ingredients of the standard medium used for culturing Dictyostelium cells were dissolved in DSW HL5dsw or ultrapure water HL5upw, and both media were autoclaved. The media were used for culturing the cells and were spiked with blasticidin $S$ for selecting the transformants. On using the extrachromosomal vector, pHK12bla, the number of isolated transformants in HL5dsw was observed to be approximately 2.7 -fold greater than that in HL5upw (fig. 1).

The gene $g b f A$ encodes the transcription factor $\mathrm{G}$ boxbinding factor, which is important for cell aggregation.

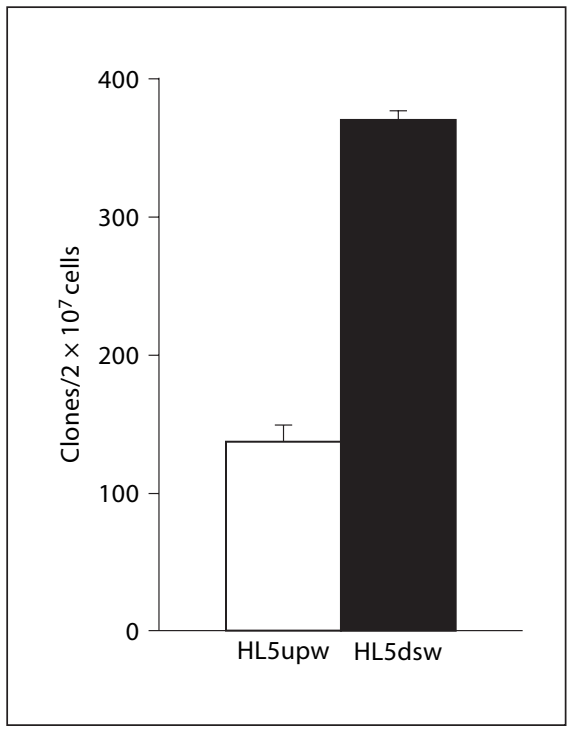

Fig. 1. Increase in the transformation efficiency of an extrachromosomal vector by DSW. The transformation efficiencies were examined using an extrachromosomal vector, pHK12bla. The data are represented as the number of primary transformants per one transformation $\left(2 \times 10^{7}\right.$ cells $)$ with the medium used being composed of either ultrapure water HL5upw (white bar) or desalted DSW HL5dsw (black bar). The bars represent the SD from three independent transformations.

Therefore, its null mutant is defective in effecting the postaggregation process [Schnitzler et al., 1994]. Both ct $x A$ and $c t x B$ genes encode cortexillin that binds actin bundles, and both are related to the process of cytokinesis occurring at the cell cortex [Faix et al., 1996]. We selected these genes for testing the effect of DSW on transformation and homologous recombination, because these genes had been very difficult to target by our previous transformation procedure using ultrapure water. On using the $g b f A$ gene disruption construct (fig. 2a), it was observed that the yield of transformants using HL5dsw was significantly increased by approximately 3.3 -fold relative to that when using HL5upw (fig. 2b). On the other hand, the transformation efficiencies for $\operatorname{ct} x A$ and $\operatorname{ct} x B$ were not increased as significantly as that of $g b f A$ but were only slightly enhanced in HL5dsw (fig. 2b). These results indicate that DSW enhanced the cellular uptake of the exogenous DNA vector, although the extent of integration of the DNA fragments into the genome varies depending on the constructs. 
Fig. 2. Increase in transformation and homologous recombination efficiencies by DSW. a The gene-targeting construct is illustrated schematically. The $b s r$ cassette was inserted into the ORF region of a genomic clone with approximately 1 or 0.7 $\mathrm{kbp}$ of the $5^{\prime}$ - and $3^{\prime}$-flanking regions for $g b f A$ or $\operatorname{ct} x A$ and $\operatorname{ct} x B(\mathbf{b})$, respectively. The transformation efficiencies were examined using the $g b f A, \operatorname{ct} x A$, and $\operatorname{ct} x B$ genetargeting constructs. The data are shown as the number of primary transformants per one transformation $\left(2 \times 10^{7}\right.$ cells $)$ with the medium used being composed of ultrapure water HL5upw (white bars) or DSW HL5dsw (black bars) (c). Homologous recombination efficiencies using the gene-targeting construct and either HL5upw or HL5dsw are represented as the average of the percentage of the gene-targeted transformants to all the transformants. The bars represent the SD from three independent transformations.
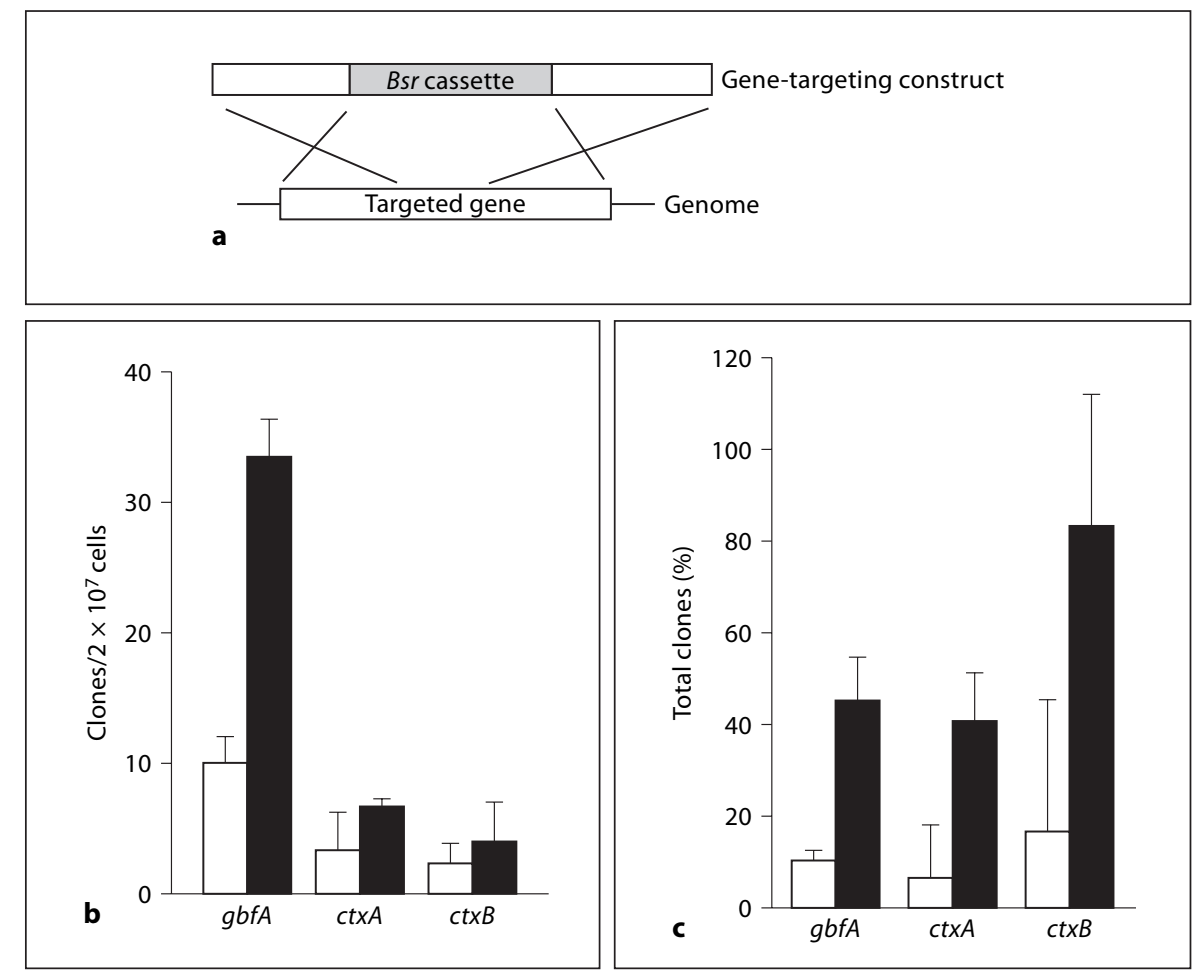

Increase in Homologous Recombination Efficiency in DSW Medium

Next, the effect of desalted DSW on homologous-recombination efficiency was investigated. During $g b f A$ gene targeting, the average homologous recombination efficiency was increased by approximately 4.5 -fold in HL5dsw (fig. 2c). The increase in the homologous recombination efficiency of $c t x A$ was comparable to that of $g b f A$. On the other hand, the homologous recombination efficiency of $\operatorname{ct} x A$ was observed to have increased more dramatically as compared to that of $\operatorname{ct} x B$. These results indicate that the DSW significantly facilitated the homologous integration of the exogenous DNA fragment into the genome.

Furthermore, considering the increase in the transformation efficiency (fig. 2b), we calculated that the average number of gene-targeted clones per one transformation was raised by approximately 15-, 8-, and 5-fold during the targeting of the genes $g b f A, \operatorname{ct} x A$, and $\operatorname{ct} x B$, respectively.

\section{Effect of Growth and Selection in DSW Medium on Transformation Efficiency}

The transformation protocol employed, using HL5 as the medium, contains two distinct steps: culturing cells (before transformation) and selecting transformants by checking for drug resistibility (after transformation). In order to determine at which step the DSW influenced the transformation efficiency, we replaced the HL5 media before and after electroporation. When the DSW medium was supplied only during the growth of transforming cells or only during the selection of transformed cells, in both cases, the transformation and homologous recombination efficiencies were significantly increased using the extrachromosomal vector pHK12bla and the gene disruption construct, respectively (fig. 3a-c). Furthermore, culturing the cells in HL5dsw prior to transformation was more influential than during the drug selection after transformation. These results indicate that culturing cells in DSW medium was more important for cellular entry and genome integration of vector DNA, than for the maintenance of transformed cells during drug selection.

\section{Growth and Cellular Recovery of Dictyostelium Cells in the DSW Medium}

The fact that DSW significantly increased the transformation and homologous recombination efficiencies could be explained by the increased recovery and growth of the electroporated cells; in HL5dsw, the cells exhibited faster growth rates in the exponential phase and reached higher cell densities in the stationary phase than in 


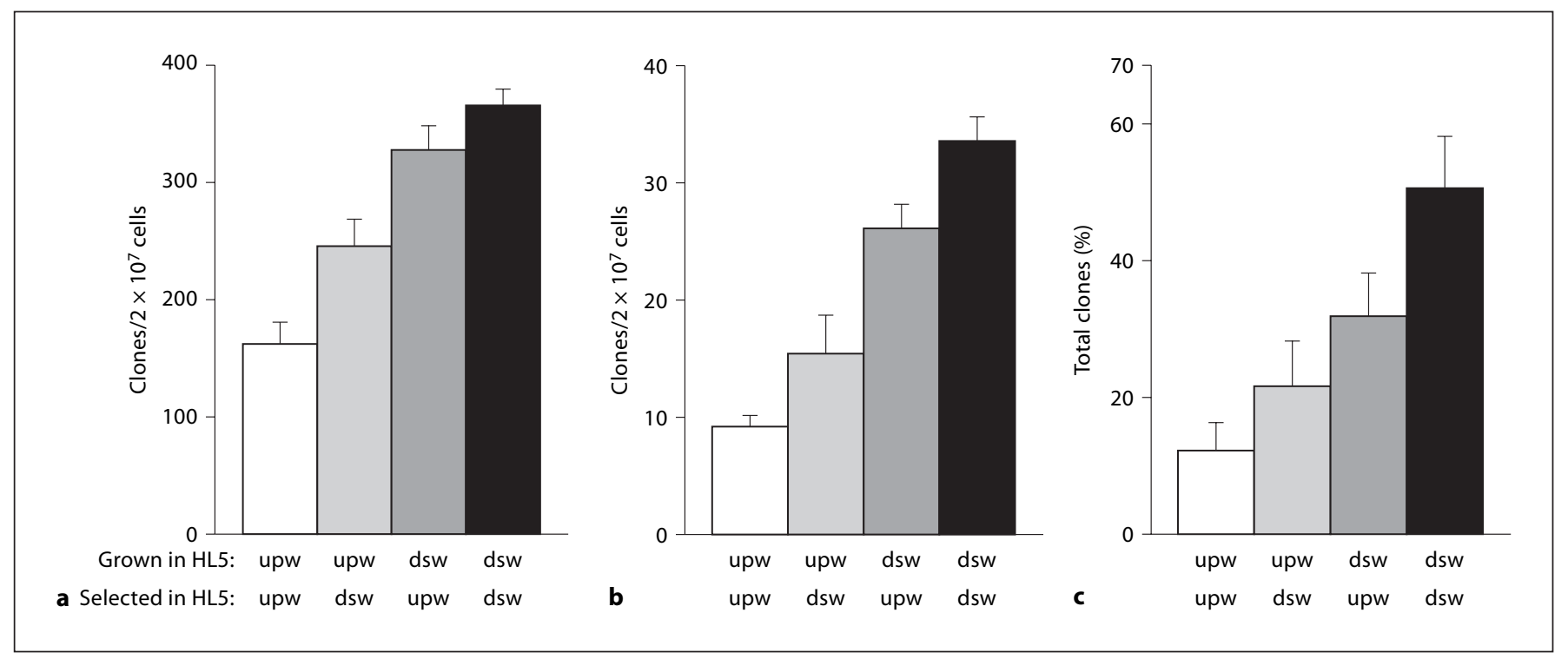

Fig. 3. The effects of DSW investigated before and after electroporation. The HL5upw and HL5dsw were either removed and replenished or left unchanged before and after transformation. The transformation efficiencies were examined using pHK12bla (a) and the gene-targeting construct for $g b f A(\mathbf{b})$. Homologous recombination efficiencies were also examined using the gene-targeting construct (c). These values are represented as the average of the percentage of the gene-targeted transformants to all the transformants. The bars represent the SD from three independent transformations.
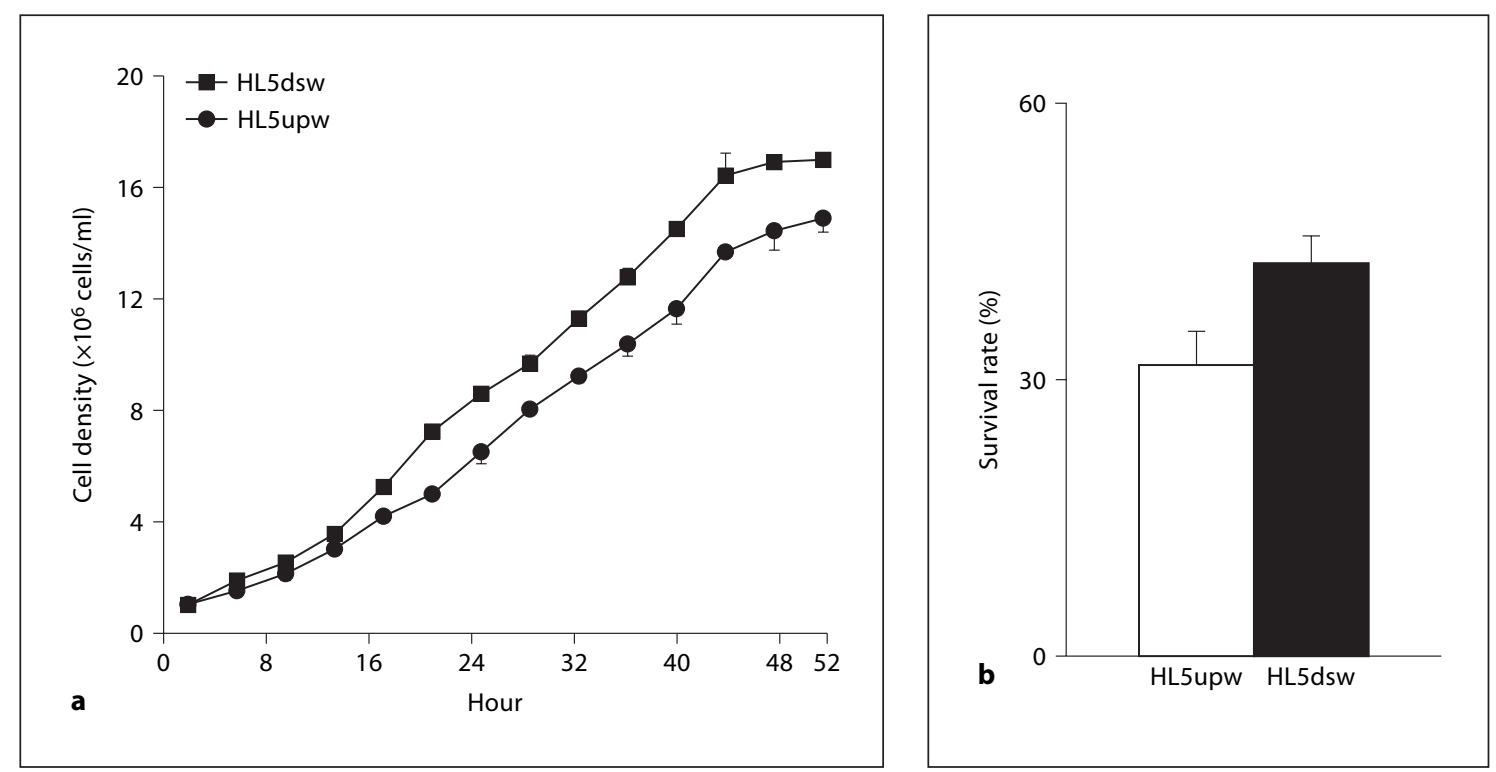

Fig. 4. Growth curve and survival rates after electroporation. a Growth of AX2 cells in HL5upw and in HL5dsw. b Survival rate is represented as the percentage of the number of primary clones to the number of electroporated cells. The bars represent the SD from three independent transformations. 
HL5upw (fig. 4a). Furthermore, the survival rate of electroporated cells in HL5dsw was also improved by approximately 1.3 -fold (fig. 4b). These results suggest that the increase in transformation efficiency caused by DSW was partially due to the improved growth and survival rates.

\section{Discussion}

In this study, the positive effects of using DSW as a solvent medium on transformation were reported. It is generally acknowledged that both the exponential growth of cells before electroporation and good initial growth condition after electroporation are important for high efficiency. Thus, it is presumed that the small difference in growth rate observed in figure 4 a would be sufficient to facilitate an increase in transformation efficiency. However, prolonged incubation of the electroporated cells in the HL5upw selection medium did not increase the number of transformants (data not shown). Therefore, it is presumed that DSW influences the transformation efficiency directly, rather than via growth. One explanation for this may be that DSW contains factors that enhance the uptake of DNA and homologous recombination; the cations that are dissolved in DSW are potentially good candidates. The DSW used in this study is desalted water; however, it contains approximately $2.4 \mathrm{mM} \mathrm{Na}^{+}$ions, 0.6 $\mathrm{mM} \mathrm{Ca}^{2+}$ ions, $0.7 \mathrm{mM} \mathrm{K}^{+}$ion, and $2.4 \mathrm{mM} \mathrm{Mg}^{2+}$ ions (calculations are based on the manufacturer's data; http:// www.marine-gold.co.jp/shop/marinegold300/index. html). However, the addition of the above-mentioned concentrations of these minerals, such as chlorinated salt, to HL5upw did not increase the transformation efficiency when the extrachromosomal vector, pHK12bla (data not shown) was used. This result indicates that an unidentified minor factor(s), possibly cell permeable and DNA-binding, is likely to be the key mediator of the enhanced transformation and homologous recombination efficiencies.

We used an ECM 830 electroporator (BTX) and conducted 15 successive square pulse electroporations. This may be a rather unusual condition for Dictyostelium transformation because gene pulsers (Bio-Rad) are the standard equipment used for electroporation in most laboratories that conduct Dictyostelium research. We have used a gene pulser with a standard Dictyostelium transformation protocol and confirmed that there was an effective increase in the transformation efficiency when the extrachromosomal vector and the gene-targeting constructs were used (data not shown).

DSW Increases Transformation and Homologous Recombination Efficiencies
The use of DSW raised the yield of transformants in other Dictyostelium strains (KAX3, AX4 - data not shown) and in a non-axenic strain, V12M2, when used in conjunction with different gene-targeting constructs [Muramoto and Urushihara, 2006]. Furthermore, during random mutagenesis employing random restriction enzyme-mediated integration, we observed the upregulation of the yield and variation in the primary transformants (data not shown).

\section{Experimental Procedures}

\section{Materials}

DSW, Marine Gold 300, was supplied by the Marine Gold Corp. (Muroto City, Kochi, Japan), type I ultrapure water was obtained from Millipore Corp. (Billerica, Mass., USA), and blasticidin $S$ was purchased from Funakoshi Corp. (Tokyo, Japan).

\section{Cell Culture and Transformation}

The HL5 medium for culturing $D$. discoideum AX2 cells was prepared by dissolving $15.4 \mathrm{~g}$ glucose, $7.15 \mathrm{~g}$ Difco yeast extract, $14.3 \mathrm{~g}$ Difco proteose peptone, $0.485 \mathrm{~g} \mathrm{KH}_{2} \mathrm{PO}_{4}$, and $1.28 \mathrm{~g}$ $\mathrm{Na}_{2} \mathrm{HPO}_{4} \cdot 12 \mathrm{H}_{2} \mathrm{O}$ in either the DSW (Marine Gold 300, HL5dsw) or the type I ultrapure water HL5upw obtained by using the Millipore filtration system. For the measurement of growth rate, the cells were inoculated at a density of $1.0 \times 10^{6}$ cells $/ \mathrm{ml}$ in $20 \mathrm{ml}$ HL5upw or HL5dsw and shaken in a 100-ml flask at $120 \mathrm{rpm}$ at $21^{\circ} \mathrm{C}$. Electroporation was carried out using an ECM 830 square pulse electroporator (BTX) using $400 \mu \mathrm{l}$ of $5 \times 10^{7}$ cells in electroporation buffer $\left(10 \mathrm{~mm} \mathrm{NaPO}_{4}, 50 \mathrm{~mm}\right.$ sucrose, $\mathrm{pH}$ 6.1, in ultrapure water) in a $2-\mathrm{mm}$ gapped cuvette under the following conditions: $500 \mathrm{~V}, 100 \mu \mathrm{s}, 15$ times with 1-s intervals [Kuwayama et al., 2002]. Either $1 \mu \mathrm{g}$ of the extrachromosomal vector, pHK12bla, which contains a multicloning site in the MB12n plasmid [Linskens et al., 1999] or $10 \mu \mathrm{g}$ of gene-targeting construct for $g b f A$, $\operatorname{ct} x A$, and $\operatorname{ctx} B$ was used. Following electroporation, the cell suspension was incubated at $21^{\circ} \mathrm{C}$ for 15 min with $4 \mu \mathrm{l}$ of $100 \mathrm{mM}$ $\mathrm{CaCl}_{2}$ and $\mathrm{MgCl}_{2}$, and then $40 \mathrm{ml}$ of HL5upw or HL5dsw was added to the electroporated cell suspension. In order to ensure the clonal isolation of the primary transformants and to evaluate the transformation efficiency, the cell suspension was immediately divided into 100- $\mu$ l aliquots and dispensed into the wells of four 96-well culture dishes. The dishes were incubated for $24 \mathrm{~h}$ at $21^{\circ} \mathrm{C}$ followed by the addition of $100 \mu \mathrm{l}$ of $20 \mu \mathrm{g} / \mathrm{ml}$ blasticidin S. The dishes were incubated for a further 1 week until the clones became detectable as cell spots in each well. For measuring the survival rate, the electroporated cell suspension containing $1 \mu \mathrm{g}$ of the extrachromosomal vector was diluted 107 -fold by successive dilutions with HL5upw or HL5dsw and a 100- $\mu$ l aliquot that did not contain the selective drug was further divided and dispensed into four 96 -well dishes at $21^{\circ} \mathrm{C}$ for 1 week. In $g b f A$, gene disruption was examined by the phenotypic assessment of primary transformants; this was performed by plating the isolated clones on a $5 \mathrm{LP}$ agar plate $(0.5 \%$ lactose, $0.5 \%$ Bacto Peptone and $1.5 \%$ Difco agar) with Escherichia coli $\mathrm{B} / \mathrm{r}$ as the food source. 
Gene Disruption Construct and PCR

The gene disruption construct for $g b f A$ (Entrez Gene ID 3388141) was synthesized by a polymerase chain reaction (PCR)dependent technique [Kuwayama et al., 2002]. Briefly, the 5'flanking region of the construct was amplified with two primers, 5'-CATGAGTAACTCAAATAATAATAG-3' and 5'-GTAATCATGGTCATAGCTGTTTCCTGCAGCTGCTTCATTACAACGAG-3'. The 3'-flanking region of the construct was amplified with primers, $5^{\prime}$-CCTTGAAAAGATGTGCATATGGAT-3' and 5'-CACTGGCCGTCGTTTTACAACGTCGACTATTACCAAAATGCAACAAG-3'. The $b s r$ cassette in the multicloning site of pUCBsr $\Delta$ Bam [Adachi et al., 1994] was amplified using the primer pair 5'-CTGCAGGAAACAGCTATGACCATGATTAC-

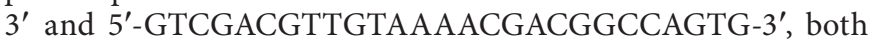
of which are complementary to the two underlined regions, respectively. The three amplified fragments were subjected to fusion PCR that produced the required gene-targeting construct (fig. 2a). The gene-targeting constructs were cloned using a TOPO TA cloning kit for sequencing (Invitrogen Corp., Calif., USA).

The genomic regions of ctxA (Entrez Gene ID 3387750) and ctxB (Entrez Gene ID 3394130) open reading frames (ORFs) were obtained by PCR amplifications using the primers 5'-ATGGCAGGTAAAGATTGGG-3' and 5'-TTATTTTTTTGATTTTGATG$3^{\prime}$ for $\operatorname{ct} x A$ and the primers $5^{\prime}$-ATGGATTTAAATAAAGAATG- $3^{\prime}$ and 5'-TTATTTTTTAGCAGCAGCTT-3' for $c t x B$. The PCR fragments were cloned with the TOPO TA cloning kit for sequencing, and the $b s r$ cassette was inserted into $M f e I$ site at the 655th bp from the $5^{\prime}$-end of the $c t x A$ genomic clone and HindIII site at the 721st bp from the $5^{\prime}$-end of the $c t x B$ genomic clone.

During transformation, the gene-targeting constructs were amplified by PCR using up to $10 \mu \mathrm{g}$ of the outermost primers for one transformation and the cloned vectors as templates. The gene disruption of $\operatorname{ct} x A$ was confirmed by PCR using primers designed to be homologous to the $b s r$ gene (5'-AAGTAGCGACAGAGAAGATT-3' for $c t x A$ and $5^{\prime}$-GGGTATATTTGAGTGGAATG-3' for $\operatorname{ct} x B$ ) and downstream of the $3^{\prime}$-end of the $\operatorname{ct} x A$ ( $5^{\prime}$-AATTAGGTTATCATTGTACAAG-3' for $\operatorname{ct} x A$ ) or $\operatorname{ct} x B$ (5'-AATAATGGCCATTATTGAGGG-3' for $c t x B$ ) clones. The genomic DNA from each transformant was used as a template.

\section{Acknowledgement}

We thank Dr. Hideko Urushihara for her helpful comments on the study. This work was supported by the special fund for tenure-track faculty members of the Institute of Biological Sciences at the University otTsukuba.

\section{References}

Adachi H, Hasebe T, Yoshinaga K, Ohta T, Sutoh $\mathrm{K}$ : Isolation of Dictyostelium discoideum cytokinesis mutants by restriction enzymemediated integration of blasticidin $\mathrm{S}$ resistance marker. Biochem Biophys Res Commun 1994;205:1808-1814.

Alibaud L, Cosson P, Benghezal M: Dictyostelium discoideum transformation by oscillating electric field electroporation. Biotechniques 2003;35:78-80, 82-83.

Betapudi V, Shoebotham K, Egelhoff TT: Generation of double gene disruptions in Dictyostelium discoideum using a single antibiotic marker selection. Biotechniques 2004;36: 106-112.

Eichinger L, Pachebat JA, Glockner MA, et al: The genome of the social amoeba Dictyostelium discoideum. Nature 2005;435:43-57.

Faix J, Steinmetz M, Boves H, Kammerer RA, Lottspeich F, Mintert U, Murphy J, Stock A, Aebi U, Gerisch G: Cortexillins, major determinants of cell shape and size, are actin-bundling proteins with a parallel coiled-coil tail. Cell 1996;86:631-642.
Khanna M, Stotzky G: Transformation of Bacillus subtilis by DNA bound on montmorillonite and effect of DNase on the transforming ability of bound DNA. Appl Environ Microbiol 1992;58:1930-1939.

Kessin RH: Dictyostelium Evolution, Cell Biology, and the Development of Multicellularity. Cambridge University Press, Cambridge 2001.

Knecht D, Pang KM: Electroporation of Dictyostelium discoideum. Methods Mol Biol 1995; 47:321-330.

Kuspa A, Loomis WF: Tagging developmental genes in Dictyostelium by restriction enzyme-mediated integration of plasmid DNA. Proc Natl Acad Sci USA 1992;89:88038807.

Kuwayama H, Obara S, Morio T, Katoh M, Urushihara H, Tanaka Y: PCR-mediated generation of a gene disruption construct without the use of DNA ligase and plasmid vectors. Nucleic Acids Res 2002;30:e2.
Linskens MH, Grootenhuis PD, Blaauw M, Huisman-de Winkel B, Van Ravestein A, Van Haastert PJM, Heikoop JC: Random mutagenesis and screening of complex glycoproteins: expression of human gonadotropins in Dictyostelium discoideum. FASEB J 1999;13: 639-645.

Muramoto T, Urushihara H: Small GTPase RacF2 affects sexual cell fusion and asexual development in Dictyostelium discoideum through the regulation of cell adhesion. Dev Growth Differ 2006;48:199-208.

Nellen W, Saur U: Cell-cycle dependent transformation competence in Dictyostelium discoideum. Biochem Biophys Res Commun 1988;154:54-59.

-Schnitzler GR, Fischer WH, Firtel RA: Cloning and characterization of the G-box-binding factor, an essential component of the developmental switch between early and late development in Dictyostelium. Genes Dev 1994;8:502-514. 